\title{
Differential diagnosis of thyroid nodules using fine-needle aspiration cytology and oncogene mutation screening: are we ready?
} Rosa Marina Melillo ${ }^{1,2}$, Massimo Santoro ${ }^{1}$ and Giancarlo Vecchio ${ }^{1 *}$

Addresses: ${ }^{1}$ Dipartimento di Biologia e Patologia Cellulare e Molecolare 'L. Califano' (DBPCM), University of Naples Federico II, Via S. Pansini, 5 , 80131 Naples, Italy; ${ }^{2}$ Istituto per l'Endocrinologia e l'Oncologia Sperimentale del CNR 'G. Salvatore' (IEOS), Via S. Pansini, 5, 80131 Naples, Italy

*Corresponding author: Giancarlo Vecchio (vecchio@unina.it)

FI000 Medicine Reports 2010, 2:62 (doi:10.3410/M2-62)

The electronic version of this article is the complete one and can be found at: http://fl000.com/reports/medicine/content/2/62

\begin{abstract}
Thyroid nodules are a very common clinical finding, and although the majority of them are benign, thyroid carcinoma accounts for about $5-15 \%$ of nodules. Fine-needle aspiration cytology (FNAC) is actually used for the differential diagnosis of these lesions. Although in most cases this examination clearly distinguishes benign from malignant lesions, some fine-needle aspiration (FNA) samples fall into undetermined thyroid cytology categories, which according to the most recent classification of thyroid FNAC consist of 'suspicious for malignancy', 'suspicious for follicular or Hurtle cell neoplasm', and 'follicular lesion of undetermined significance/atypia of undetermined significance'. Moreover, some samples are insufficient for diagnosis. Taken together, these categories account for almost $20-30 \%$ of nodules. Owing to the high risk of papillary thyroid carcinoma, patients with lesions that are 'suspicious for malignancy' are currently subjected to lobectomy or total thyroidectomy. On the other hand, patients with 'atypia of undetermined significance' undergo repeated FNAs, and patients with 'suspicious for follicular or Hurtle cell neoplasm' are subjected to diagnostic lobectomy and subsequently, in the case of histological diagnosis of carcinoma, total thyroidectomy. Recent studies clearly indicate that molecular analysis of thyroid nodules can significantly improve the diagnostic power of cytology and drive the appropriate clinical management of these patients.
\end{abstract}

\section{Introduction and context}

The incidence of thyroid nodules detected by clinical examination or by ultrasonography is very high in the adult population. For establishing whether these lesions are benign or malignant, fine-needle aspiration cytology (FNAC) is currently the gold standard. Prior to the routine use of thyroid fine-needle aspiration (FNA), only $14 \%$ of surgically resected thyroid nodules were found to be malignant [1]. With current thyroid FNA practice, greater than $50 \%$ of resected nodules are malignant [2]. Benign lesions identified by FNAC are generally left untreated, but patients undergo periodic clinical and ultrasound examination. Patients with malignant nodules undergo total thyroidectomy. However, FNAC is hampered by some limitations. First, some FNA samples (non-diagnostic) are simply insufficient for the diagnosis because they contain only cystic fluid or scant material, and this requires that patients' repeat FNA with ultrasound guide. Second, some FNACs reveal lesions of uncertain nature. These lesions have been classified according to the suggested thyroid FNA classification scheme of the National Cancer Institute [3] using the following categories: 'suspicious for malignancy', 'suspicious for follicular neoplasm', 'suspicious for Hurtle cell neoplasm', and '[follicular] lesions of undetermined significance (FLUSs)/atypia of undetermined significance'. The 'suspicious for malignancy' category includes entities for which the evidence for malignancy is not definitive. It represents 3-9\% of all thyroid FNA results, and between $60-77 \%$ of these cases prove to be malignant (mainly papillary thyroid carcinoma, or PTC) [2,4-6]. The 'suspicious for follicular cell neoplasm' 
group is particularly heterogenous and includes lesions with significant architectural atypia and follicular proliferation. These lesions include follicular adenoma (FA), follicular thyroid carcinoma (FTC), and follicular variant of PTC (fvPTC). Because it is not possible to identify capsular or vascular invasion (considered to be the hallmarks of FTC), a definitive diagnosis of follicular carcinoma by FNA cannot be made. For this reason, patients who present with these features must undergo diagnostic lobectomy. The malignancy rate for these cases ranges from $14 \%$ to $32 \%[2,4-6]$. The 'suspicious for Hurtle cell neoplasm' category also includes Hurtle cell carcinoma and Hurtle cell adenoma, for which the differential diagnosis on the basis of FNAC is not possible and hence a diagnostic lobectomy is necessary. Finally, the FLUS group includes cases that do not fit into any of the other categories. The malignancy rate (approximately 5-10\%) of these lesions is not sufficient to justify immediate surgery, and the recommended treatment is repeated FNAs. Taken together, these studies indicate that, although FNAC is the gold standard for the differential diagnosis of thyroid nodules, this technique has some limitations, and a certain number of lesions remain undefined. The discovery of specific genetic lesions in different histotypes of human thyroid cancer has raised the possibility of improving the diagnostic accuracy of FNAC. Thyroid cancer, though a rare disease, is the most frequent endocrine neoplasia and its incidence is rapidly increasing. Three malignant lesions derive from follicular cells: well-differentiated thyroid carcinomas (which include PTC and FTC), poorly differentiated thyroid carcinomas, and anaplastic thyroid carcinomas [7]. Non-overlapping mutations of the RET, TRKA, RAS, and BRAF genes are found in PTCs. Activating point mutations in the genes encoding RAS small GTPases are found mainly in fvPTC [8]. Point mutations in BRAF are the most common genetic lesions in PTCs [9-12]. FTCs are characterized by RAS point mutations [13] or by the PAX8/PPAR $\gamma$ rearrangement [14]. RAS point mutations can also be found in FAs [15].

In recent years, a number of studies that report the use of several techniques of mutation detection in material obtained by FNAC for the diagnosis of thyroid nodules have been published [16,17]. These studies, taken together, support the feasibility of testing the presence of mutations in FNA samples and suggest that the presence of these mutations could increase the diagnostic accuracy for thyroid nodules. However, most of these studies are retrospective, analyze BRAF status almost exclusively (and RAS, RET/PTC, and PAX8/PPAR $\gamma$ genetic alterations only in some cases), and do not provide information regarding the specificity and sensitivity of molecular analysis or its diagnostic power in comparison with cytology.

\section{Recent advances}

Recently, some studies have shown that molecular analysis of thyroid nodules can be applied in a clinical setting and can improve the accuracy of the diagnosis. In a large prospective study, Nikiforov and colleagues [18] systematically analyzed 470 FNAs from 328 patients for a panel of genetic alterations, including BRAF, RAS, RET/ PTC, and PAX8/PPAR $\gamma$ mutations, and correlated these results with cytology, surgical pathology, and clinical follow-up. The presence of a mutation was a strong indicator of malignancy, with a specificity of almost $100 \%$. BRAF, RET/PTC, and PAX8/PPAR $\gamma$ mutations were always associated with carcinomas, whereas RAS mutations were found in FA in a few cases, but never in hyperplastic nodules. However, the sensitivity of molecular testing was around 60\%. Cytology was 100\% specific in identifying malignant lesions, but its sensitivity was only $40 \%$. Together, molecular analysis and cytology reached $80 \%$ sensitivity. Thus, mutation screening was able to identify malignant lesions that could not be identified by cytology. A few cases carrying genetic alterations have been identified in the cytology-negative group, but most of the mutation-positive cases fell in the group of indeterminate cytology. Thus, molecular analysis is particularly helpful for this group. This concept is further supported by another report, in which 513 indeterminate lesions (FLUSs) were specifically analyzed [19]. For 117 of them, surgical pathology outcome was available. Twenty of them were carcinomas, according to pathological analysis. Molecular screening identified 12 cases positive for BRAF, RAS, or PAX8/PPAR $\gamma$ (no RET/ PTC mutations were found in this sample set), and all of them were carcinomas, indicating that FLUSs with positive molecular tests have a high probability of being malignant. Similar findings were reported by Cantara and colleagues [20]. These authors analyzed 235 FNA samples for BRAF, RET, TRKA, and PPAR $\gamma$ mutations and found 67 genetic alterations. The same mutations were searched for in surgical tissue samples from the same patients, in which 76 mutations were found. All of the mutations found in cytological samples were also observed in the corresponding tissue sample. Moreover, in the tissues, additional mutations were found. Molecular analysis improved the diagnostic performance of FNAC. Importantly, this report also indicates that the molecular analysis is particularly useful in samples with indeterminate or inadequate cytology. Moreover, molecular analysis identified six PTCs in the group with benign cytology [20].

In both reports, the authors analyzed the status of known oncogenes in thyroid nodules and found that molecular diagnosis could improve the diagnostic accuracy of FNAC. However, some malignant lesions could not be identified through this method. One possibility is that, 
for technical reasons, the methodological tests are not accurate. Indeed, whereas DNA retrieval from FNA is quite easy and reliable, RNA extraction from these samples is more difficult, and good quality material cannot always be obtained. This would impair the detection of RET/PTC or PAX8/PPAR $\gamma$ rearrangement in particular. So it is possible that at least some rearrangements are missed in this screening. This is indeed confirmed by the report by Cantara et al. [20]. These authors found more mutations in tissue samples than in FNACs and suggest that the discrepancy between results obtained from cytology and pathology samples was due to technical reasons and was more frequent in the RET/ PTC-positive cases [20]. Another possibility is that some thyroid carcinomas harbor rare mutations in known oncogenes that were not searched for. Moreover, since the RET/PTC-RAS-BRAF-ERK signaling pathway is relevant for PTCs [21], it is possible that other mutations may be found in novel genes that are effectors or regulators of this signaling cascade. Alternatively, other mutations could affect genes that belong to distinct signaling pathways.

Taken together, these reports strongly indicate that the molecular screening of FNA samples is feasible and useful. A standardization of the techniques is urgently needed in order for the results between the different diagnostic centers to be comparable. polymerase chain reaction on DNA and identification of mutations in $B R A F$ and RAS genes is a sensitive, specific, reliable, and reproducible technique. However, the identification of RET/PTC and PAX8/PPAR $\gamma$ rearrangements is more problematic and an amelioration of this technique or the application of a novel method should be considered. Moreover, the search for novel genes or markers of malignancy should definitely be pursued.

\section{Implications for clinical practice}

These studies are very important for the clinical management of thyroid nodules. They indicate that molecular diagnosis is more powerful than cytology in identifying true carcinomas and that, together with cytological analysis, it can significantly improve the diagnostic accuracy of thyroid nodules. This is particularly true for lesions of indeterminate nature, which are the most troublesome. In this set of lesions, the finding of a mutation was almost always associated with malignancy. This would indicate that patients who have undefined cytology but who are positive for molecular tests should undergo total thyroidectomy instead of repeated FNAs. Patients who have a cytological diagnosis 'suspicious for malignancy' and who also score positive on molecular tests should directly undergo total thyroidectomy instead of diagnostic lobectomy.
We think that not only indeterminate but also benign and malignant FNAC samples should undergo molecular analysis. Patients with indeterminate or benign cytology will benefit from molecular tests because some malignant cases not diagnosed with cytology will be identified and cured. In patients with malignant cytology, molecular analysis could improve the prognostic power of FNAC and influence therapy by identifying the specific mutation For instance, the presence of a BRAF mutation is indicative of a worse prognosis and might suggest a more radical treatment [22]. Moreover, the identification of a BRAF mutation, but not a RET/PTC or RAS mutation, predicts responsiveness to mitogen-activated protein kinase kinase (MEK) inhibitors [21,23]. In the reports discussed above, some cases that scored positive for RAS mutation turned out to be FA, a benign lesion $[18,20]$. However, the authors suggest that precautional thyroidectomy in these patients would be a reasonable choice since RAS-positive FAs are considered precursors of PTC or FTC and since RAS mutations are also associated with a worse prognosis in thyroid cancer [24]. We agree with this view and suggest that RAS-positive lesions be treated with total thyroidectomy, irrespectively of the cytological or pathological diagnosis.

\section{Abbreviations}

FA, follicular adenoma; FLUS, follicular lesion of undetermined significance; FNA, fine-needle aspiration; FNAC, fine-needle aspiration cytology; FTC, follicular thyroid carcinoma; fvPTC, follicular variant of papillary thyroid carcinoma; PTC, papillary thyroid carcinoma.

\section{Competing interests}

The authors declare that they have no competing interests.

\section{Acknowledgments}

Our research at DBPCM and IEOS is supported by the Associazione Italiana per la Ricerca sul Cancro (AIRC), the Italian Ministry of University and Research (MIUR), the Italian Ministry of Health, and the Istituto Superiore di Oncologia (ISO).

\section{References}

I. Hamberger B, Gharib H, Melton LJ 3rd, Goellner JR, Zinsmeister AR: Fine-needle aspiration biopsy of thyroid nodules. Impact on thyroid practice and cost of care. Am J Med 1982, 73:38I-4.

2. Yassa L, Cibas ES, Benson CB, Frates MC, Doubilet PM, Gawande AA, Moore FD Jr, Kim BW, Nosé V, Marqusee E, Larsen PR, Alexander EK: Long-term assessment of a multidisciplinary approach to thyroid nodule diagnostic evaluation. Cancer 2007, I I I:508- I6.

3. Layfield LJ, Cibas ES, Gharib H, Mandel SJ: Thyroid aspiration cytology: current status. CA Cancer J Clin 2009, 59:99-I I0.

4. Gharib H, Goellner JR, Johnson DA: Fine-needle aspiration cytology of the thyroid. A I2-year experience with II,000 biopsies. Clin Lab Med 1993, 13:699-709. 
5. Logani S, Gupta PK, LiVolsi VA, Mandel S, Baloch ZW: Thyroid nodules with FNA cytology suspicious for follicular variant of papillary thyroid carcinoma: follow-up and management. Diagn Cytopathol 2000, 23:380-5.

6. Yang J, Schnadig V, Logrono R, Wasserman PG: Fine-needle aspiration of thyroid nodules: a study of $\mathbf{4 7 0 3}$ patients with histologic and clinical correlations. Cancer 2007, I I I:306- I5

7. Kondo T, Ezzat S, Asa SL: Pathogenetic mechanisms in thyroid follicular-cell neoplasia. Nat Rev Cancer 2006, 6:292-306.

8. Zhu Z, Gandhi M, Nikiforova MN, Fischer AH, Nikiforov YE: Molecular profile and clinical-pathologic features of the follicular variant of papillary thyroid carcinoma. An unusually high prevalence of ras mutations. Am J Clin Pathol 2003, I 20:7 I-7.

9. Kimura ET, Nikiforova MN, Zhu Z, Knauf JA, Nikiforov YE, Fagin JA: High prevalence of BRAF mutations in thyroid cancer: genetic evidence for constitutive activation of the RET/ PTC-RAS-BRAF signaling pathway in papillary thyroid carcinoma. Cancer Res 2003, 63:1454-7.

10. Xu X, Quiros RM, Gattuso P, Ain KB, Prinz RA: High prevalence of BRAF gene mutation in papillary thyroid carcinomas and thyroid tumor cell lines. Cancer Res 2003, 63:456I-7.

II. Soares P, Trovisco V, Rocha AS, Lima J, Castro P, Preto A, Maximo V, Botelho T, Seruca R, Sobrinho-Simoes M: BRAF mutations and RET/PTC rearrangements are alternative events in the etiopathogenesis of PTC. Oncogene 2003, 22:4578-80.

12. Cohen Y, Xing M, Mambo E, Guo Z, Wu G, Trink B, Beller U, Westra WH, Ladenson PW, Sidransky D: BRAF mutation in papillary thyroid carcinoma. J Natl Cancer Inst 2003, 95:625-7.

13. Nikiforova MN, Lynch RA, Biddinger PW, Alexander EK, Dorn GW 2nd, Tallini G, Kroll TG, Nikiforov YE: RAS point mutations and PAX8-PPAR gamma rearrangement in thyroid tumors: evidence for distinct molecular pathways in thyroid follicular carcinoma. J Clin Endocrinol Metab 2003, 88:23I8-26.

14. Kroll TG, Sarraf P, Pecciarini L, Chen CJ, Mueller E, Spiegelman BM, Fletcher JA: PAX8-PPARgammal fusion oncogene in human thyroid carcinoma. Science 2000, 289:1357-60.

15. Lemoine NR, Mayall ES, Wyllie FS, Williams ED, Goyns M, Stringer B, Wynford-Thomas D: High frequency of ras oncogene activation in all stages of human thyroid tumorigenesis. Oncogene 1989, 4:159-64.

16. Nikiforova MN, Nikiforov YE: Molecular diagnostics and predictors in thyroid cancer. Thyroid 2009, 19:|35|-61.
17. Eszlinger M, Paschke R: Molecular fine-needle aspiration biopsy diagnosis of thyroid nodules by tumor specific mutations and gene expression patterns. Mol Cell Endocrinol 2010, 322:29-37.

18. Nikiforov YE, Steward DL, Robinson-Smith TM, Haugen BR, Klopper JP, Zhu Z, Fagin JA, Falciglia M, Weber K, Nikiforova MN: Molecular testing for mutations in improving the fine-needle aspiration diagnosis of thyroid nodules. J Clin Endocrinol Metab 2009, 94:2092-8.

FI000 Factor 3.0 Recommended

Evaluated by Jerome Hershman 06 Nov 2009

19. Ohori NP, Nikiforova MN, Schoedel KE, LeBeau SO, Hodak SP, Seethala RR, Carty SE, Ogilvie JB, Yip L, Nikiforov YE: Contribution of molecular testing to thyroid fine-needle aspiration cytology of 'follicular lesion of undetermined significance/ atypia of undetermined significance'. Cancer Cytopathol 2010, I 18:17-23.

20. Cantara S, Capezzone M, Marchisotta S, Capuano S, Busonero G, Toti P, Di Santo A, Caruso G, Carli AF, Brilli L, Montanaro A, Pacini F: Impact of proto-oncogene mutation detection in cytological specimens from thyroid nodules improves the diagnostic accuracy of cytology. J Clin Endocrinol Metab 2010, 95:1365-9.

FI000 Factor 6.0 Must Read

Evaluated by Jerome Hershman 18 Mar 2010

21. Knauf JA, Fagin JA: Role of MAPK pathway oncoproteins in thyroid cancer pathogenesis and as drug targets. Curr Opin Cell Biol 2009, $21: 296-303$.

22. Groussin L, Fagin JA: Significance of BRAF mutations in papillary thyroid carcinoma: prognostic and therapeutic implications. Nat Clin Pract Endocrinol Metab 2006, 2: I80-I.

23. Leboeuf $R$, Baumgartner JE, Benezra M, Malaguarnera R, Solit $D$, Pratilas CA, Rosen N, Knauf JA, Fagin JA: BRAFV600E mutation is associated with preferential sensitivity to mitogen-activated protein kinase kinase inhibition in thyroid cancer cell lines. J Clin Endocrinol Metab 2008, 93:2194-201.

24. Knauf JA, Ouyang B, Knudsen ES, Fukasawa K, Babcock G, Fagin JA: Oncogenic RAS induces accelerated transition through G2/M and promotes defects in the G2 DNA damage and mitotic spindle checkpoints. J Biol Chem 2006, 28 I:3800-9. 